\title{
Coronavirus disease 19 and parasitic diseases
}

\author{
Gilberto Bastidas ${ }^{1,2 *}$, María Báez², Geraldine Bastidas-Delgado ${ }^{3}$, and Daniel Bastidas ${ }^{3}$ \\ ${ }^{1}$ Center for Medical and Biotechnological Research; ${ }^{2}$ Department of Public Health Faculty of Health Sciences; ${ }^{3}$ Medical School. Faculty of Health \\ Sciences, University of Carabobo, Carabobo, Venezuela
}

\section{Dear Editor}

The regions where some parasitic diseases are endemic which are affected with coronavirus disease (COVID)-19 must be sanitarily prepared, because of this pandemic. All of them have the capacity to undermine the health efforts that governments maintain to keep parasitic endemics under control. The public health emergency generated by the COVID-19 pandemic in relation to parasitosis, undoubtedly translates into a significant reduction in the number of parasitized individuals seeking medical attention, perhaps motivated by the fear of contracting pandemic disease in health centers, as well as the neglect of those responsible for administering control programs, since their health-care efforts are aimed at treating the pandemic, factors that hinder the early diagnosis of parasitic diseases and their timely treatment ${ }^{1}$.

Furthermore, the first symptoms of COVID-19 (fever, myalgia, and fatigue) are usually indistinguishable from those observed in some parasitic diseases such as malaria, becoming a distracting factor in the clinical diagnosis of this. Health caregivers in the face of these symptoms will direct their research first toward the diagnosis of the new outbreak of infectious disease, as it is the most recent and most worrying public health problem. Later, they will direct their clinical suspicion toward malaria, with the understanding that its transmission is less vertiginous than that, which characterizes the severe acute respiratory syndrome coronavirus
(SARS-CoV)-2 coronavirus. Furthermore, the strategies for controlling the transmission of some parasitic diseases in regions where COVID-19 has arrived may be diminished or disappear completely. An example of this experience but with another virus was those lived in Guinea, Liberia, and Sierra Leone; because during the period, in which they were affected by Ebola, there was a cessation in the distribution of insecticide-treated bed nets as a strategy to intercept the malaria-transmitting mosquito, consequently, these regions experienced increases in morbidity due to this parasitosis ${ }^{2,3}$.

In addition, the synergy between the human immunodeficiency virus (HIV)/acquired immunodeficiency syndrome and tuberculosis (the first is an emerging disease and the second is an old one) can also occur between COVID-19 and some parasitic diseases, such as malaria and Chagas, thus promoting the progression of both diseases and increasing the probability of death in this coepidemic twin in their ability to generate deaths. The overlap between parasitic diseases and other infections leads to bidirectional alterations in the natural history, diagnosis, and patient response to treatment. The literature shows that HIV-infected patients with malaria, leishmaniasis, trypanosomiasis, Chagas disease, schistosomiasis, onchocerciasis, lymphatic filariasis, and intestinal helminths (ascariasis and trichuriasis, among others) have twice the number of episodes of symptomatic parasitemia. Therefore, it can be assumed that this may happen in people

\section{Correspondence:}

*Gilberto Bastidas

E-mail: bastidasprotozoo@ hotmail.com
Available online: $30-10-2020$

Date of reception: 08-06-2020

Date of acceptance: 14-07-2020 DOI: 10.24875/RMU.20000049
Medicina Universitaria. 2020;22(3):136-137 www.medicinauniversitaria.org (http://creativecommons.org/licenses/by-nc-nd/4.0/). 
affected with COVID-19 in endemic regions for diseases of this type $e^{4,5}$.

Countries with endemic parasitoses facing COVID-19 should implement actions to contain and mitigate the viral pandemic without neglecting the programs for controlling parasitic endemics. In this sense, the experience can be used, saving the sociocultural differences, of countries that have successfully faced both health problems under the rigorous monitoring and advice of the World Health Organization. Likewise, governments should make efforts to optimize the allocation of resources (with political will and unity of effort), with a view to the example of malaria in endemic and hyperendemic areas with the COVID-19 epidemic, to the massive administration of anti-Plasmodium spp. drugs. Furthermore, distribution of mosquito nets impregnated with insecticides and inclusion of diagnostic tests for malaria in febrile reactions and in suspected cases of COVID-19, these measures help in the management of COVID-19 in the understanding that they reduce the pressure on health-care personnel and substantially subjugate the clinical elements of diagnostic confusion between both diseases ${ }^{1,3,4}$.

To conclude, it is pointed out that this manuscript was conceived with the purpose of exposing the critical problems related to the possibility of simultaneous infection between COVID-19 and some parasitic diseases. This may serve first as a wake-up call, then as a resource for policy formulation, plans, and programs for the sanitary approach of such a particular interaction, in a globally interconnected world. In this sense, there are several aspects that must be borne in mind: that the morbidity of parasitoses may increase due to the lower attendance of those affected to health centers for fear of becoming infected with the SARSCoV-2 coronavirus; the neglect of parasite control programs by health personnel now focused on combating the emerging pandemic; the cessation of transmission control strategies; the similarity of symptoms between both conditions, viral and parasitic, makes diagnosis difficult; and the synergy between viral and parasitic infection that favors the progression of both.

\section{Conflicts of interest}

The authors declare no conflicts of interest.

\section{Financing}

The present investigation has not received specific grants from public sector agencies, the commercial sector, or non-profit entities.

\section{Ethical disclosures}

Protection of human and animal subjects. The authors declare that no experiments have been conducted on humans or animals for this research.

Confidentiality of data. The authors declare that they have followed the protocols of their work center on the publication of patient data.

Right to privacy and informed consent. The authors declare that patient data do not appear in this article.

\section{References}

1. World Health Organization. Coronavirus Disease 2019 (COVID-19) Situation Report-52. Geneva: World Health Organization; 2020. Available from: https:// www.who.int/docs/default-source/coronaviruse/situation-reports/20200312-sitrep-52-covid19.pdf?sfvrsn=e2bfc9c0_4. [Last accessed on 2020 Jun 01].

2. Walker P, White M, Griffin J, Reynolds A, Ferguson N, Ghani A. Malaria morbidity and mortality in Ebola-affected countries caused by decreased health-care capacity, and the potential effect of mitigation strategies: a modelling analysis. Lancet Infect Dis. 2015;15:825-32.

3. Wang J, Xu C, Wong Y, He Y, Adegnika A, Kremsner P, et al. Preparedness is essential for malaria-endemic regions during the COVID-19 pandemic. Lancet. 2020;395:1094-6.

4. Harms G, Feldmeier H. HIV infection and tropical parasitic diseases deleterious interactions in both directions? Trop Med Int Health. 2002;7:479-88.

5. Mayer K, Dukes Hamilton C. Synergistic pandemics: confronting the global HIV and tuberculosis epidemics. Clin Infect Dis. 2010;50 Suppl 3:S67-0. 